\title{
Male biological clock possibly linked to autism, other disorders
}

Over the last few years, epidemiological evidence has suggested that as men age their odds of having a child with autism, schizophrenia or bipolar disorder might increase. The findings-along with more recent genetic data-have led researchers to ask whether the mutations that accumulate in sperm DNA with age might underlie this observed association.

"If this paternal age effect has something to do with mutations, then that opens up all sorts of interesting and sort of scary possibilities," says Jonathan Sebat, a human geneticist at Cold Spring Harbor Laboratory in New York State. He says it is conceivable that the trend of delaying fatherhood might contribute to an increased incidence of mutations in the population that can give rise to neuropsychiatric disorders.

In a study of more than 100,000 people, along with records about their parents' ages, Avi Reichenberg at King's College London and his colleagues found that 33 out of every

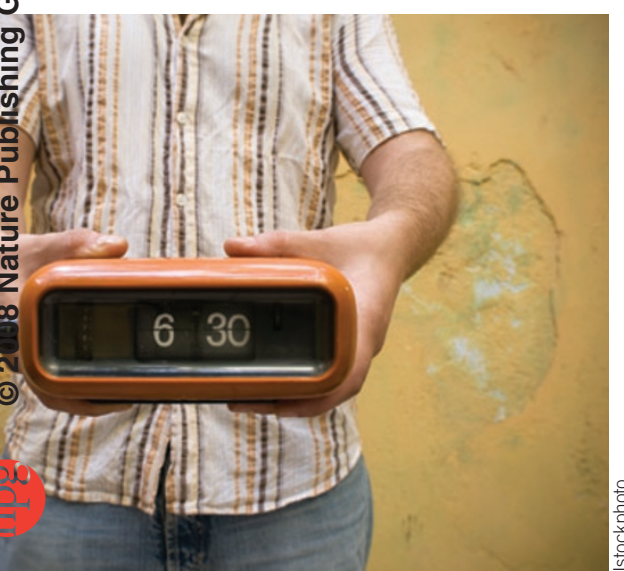

Time for fatherhood: Aging affects sperm
10,000 offspring of men 40 years or older had autism spectrum disorder-a $475 \%$ increase compared to offspring of men younger than 30 , who fathered afflicted children at a rate of 6 per 10,000 (Arch. Gen. Psychiatry 63, 10261032; 2006). This association is now being tested in a larger study, says Reichenberg. A study this September showed a similar but less pronounced association of parental age with bipolar disorder (Arch. Gen. Psychiatry 65,1034-1040; 2008).

Spontaneous mutations can arise in both sperm and eggs. As women age, for example, they have an increased risk of delivering a child with Down's syndrome and other disorders caused by large-scale chromosome problems in eggs, such as trisomy. But unlike eggs, sperm arise from stem cells that continuously divide-about 840 times by the time a man is 50 years old (Cytogenet. Genome Res. 111, $213-228 ; 2005)$. The theory is that the chances of mutations increase with each round of DNA replication-a process that could underlie estimates that the mutation rate in males is about five times that in females (Nature 416, 624-626; 2002).

"Any mutation you can think of occurs more frequently in the sperm of older men," says Sebat.

Meanwhile, recent genetic surveys of people with autism and other neuropsychiatric disorders have bolstered this controversialand still tenuous-hypothesis. The DNA studies have suggested that 'spontaneous' mutations contribute to schizophrenia and autism. This type of mutation can arise in the sperm or egg of the parents.

Sebat and his colleagues, for instance, looked at spontaneous deletions and duplications measuring about 100,000 DNA base pairs and longer-a length that often contain dozens of genes-in the genome of people with of autism spectrum disorders (Science 316, 445-449; 2007). Such spontaneous mutations occurred in only $1 \%$ of unaffected people, but they occurred in about $10 \%$ of subjects with sporadic forms of the disorder, meaning they had no family history. The researchers' methods only pick up a fraction of mutations, so the effect of sporadic mutations is probably substantially larger, says Sebat.

Similar studies this year have shown that people with nonfamilial forms of schizophrenia also have a higher rate of spontaneous duplications and deletions, and Sebat says his unpublished data show a similar association in bipolar disorder.

But whether the mutations that arise spontaneously in neuropsychiatric disorders come mainly from mom or dad is still unclear, as is their association with parental age. Sebat says larger studies underway should help clarify these questions.

And researchers caution that they have very little idea how the disrupted genes in eggs and sperm might potentially give rise to neuropsychiatric disease. "It is not established, and it can put a class of individuals in a negative light," says Rita Cantor, a human geneticist at the University of California, Los Angeles.

Moreover, other, even more tenuous explanations could underlie the parental age effect—such as the idea that fathers who delay parenthood somehow have genes that affect their social behavior and make their offspring more prone to neuropsychiatric disorders. Says Cantor, "I think it's a delicate subject."

Charlotte Schubert, Washington, DC

\section{New techniques preserve fertility hope for women}

For a man battling cancer, preserving the option to have children later in life is simple: store samples of semen. Even a single ejaculate contains millions of sperm that can later be used to fertilize an egg.

A woman facing cancer, on the other hand, has far fewer choices, which depend on her age, how much time she has before treatment must begin and the availability of a partner who can provide sperm. Oocytes, or eggs, are particularly vulnerable to chemotherapy and radiation, leaving many women infertile after being treated for cancer.

The most successful option for a woman of child-bearing age is to create embryos through in vitro fertilization and freeze them. (Even if the woman's ovaries are removed, her uterus can still carry a transplanted embryo to term.) Doctors have turned to this method for over two decades, with a success rate of up to $40 \%$. "That's a procedure that doesn't need improvement," says Kutluk Oktay, director of reproductive medicine and infertility at New York Medical College.

Women who don't have a partner can try to freeze unfertilized eggs. But, unlike hardy embryos, eggs are sensitive to chilling. Hundreds of babies have been born with this technique, but the success rate overall hovers around 3\%. "It's a big drop from embryo freezing," says Tommaso Falcone, chair of obstetrics and gynecology at the Cleveland Clinic. "[But] within the next five years, we'll solve a lot of the cryogenic challenges for freezing eggs."

Those who do have a partner who can supply sperm to create an embryo, however, might face another challenge: it takes about two weeks to stimulate the ovary to produce enough eggs for this procedure-time a woman with cancer may not have. In such cases, doctors can remove egg follicles from an ovary and bathe them in nutrients to turn them into mature eggs. This method, dubbed in vitro maturation, also has a low rate of success and carries a higher risk of miscarriage. 


\section{As IVF becomes more common, some concerns remain}

An increasing number of infertile couples have turned to assisted reproduction technology, which facilitates the union of the sperm and the egg. But, in recent years, controversial reports of birth defects in babies conceived through assisted reproduction have led a few researchers to raise concerns about the technology's safety.

Assisted reproduction includes a handful of procedures, many of which are based on in vitro fertilization (IVF) of the egg. In the US alone, assisted reproduction accounted for slightly more than $1 \%$ of all births in 2005, according to the country's Centers for Disease Control and Prevention. The IVF process poses some minor risks to women, including ovarian cysts, mood changes and hot flashes. But a few rare risks to the fetus have given some fertility researchers pause.

Tinkering with sex cells and embryos outside the body, scientists worry, might spur genetic changes that manifest as congenital birth defects. No direct evidence supports that notion, but epidemiological studies have shored up possible links between assisted reproduction and rare genetic syndromes in newborns, such as Beckwith-Wiedemann syndrome, a condition marked by premature birth, an enlarged tongue and heightened susceptibility to tumors, respiratory and speech defects (Hum. Reprod. Update 10, 3-18; 2004).

Fortunately, the syndrome is rare: it normally affects about 1 out of every 12,000 newborns worldwide. But a study found that 3 out 65 US children afflicted with the syndrome had been conceived through IVF (Am. J. Hum. Genet. $72,156-160 ; 2003)$. In other studies, 6 out of 149 children in British and French medical registries of the syndrome were found to have been conceived through IVF or through a

A more experimental option is to remove strips of ovarian tissue, freeze them, and transplant them back into the woman after treatment. Although researchers are still trying to figure out how to reconnect the tissues to the blood supply, at least four women have given birth after an ovarian transplant. This

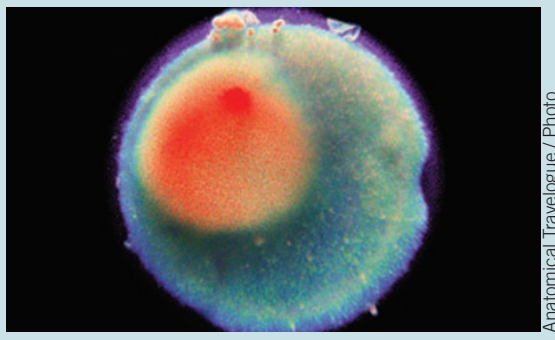

Precious commodity: The human egg specialized technique called intracytoplasmic sperm injection, or ICSI (J. Med. Genet. 40, 62-64; 2003; Am. J. Hum. Genet. 72, 1338-1341; 2003).

"There appeared to be more children with the syndrome conceived through ART [assisted reproductive technology] than we might have expected by chance alone," says Eamonn Maher, a geneticist at the University of Birmingham, UK. Many cases of Beckwith-Wiedemann syndrome, Maher says, stem from abnormalities in DNA methylation — addition of a chemical tag called a methyl group-occurring in specific genes on chromosome 11.

Methylation is one of an array of DNA markers, called imprints, that guide normal development of the embryo. For example, imprinting seems crucial to proper brain growth, says Carmen Williams, a clinical investigator at the US National Institute of Environmental Health Sciences in North Carolina. "The concern is that while the embryo is being cultured in the [IVF] lab, maybe the imprint marks are being changed. We know for sure that happens in mice," she adds (Biol. Reprod. 62, 1526-1535; 2000).

Some studies have suggested a causal relationship between ICSI and abnormal methylation patterns (Am. J. Hum. Genet. 71, 162-164; 2002; Am. J. Hum. Genet. 72, 218-219; 2003). And Maher says the findings hint that imprinting defects might trigger BeckwithWiedemann syndrome.

But he also cautions that the absolute risk of giving birth to a child with BeckwithWiedemann syndrome is low.

"The disease is so rare that it's difficult to counsel an infertile couple not to go forth with ART," Williams says.

last method also holds the greatest promise for children. "You're not going to do embryo freezing with a ten-year-old," notes Oktay.

In the pipeline are also several drugs that promise to protect ovaries from the effects of chemotherapy. In one trial, Kate Stern of the Royal Women's Hospital in Melbourne and her colleagues gave 18 women, with an average age of 25 , a drug called cetrorelix. The drug blocks gonadotropin-releasing hormone, effectively sending ovaries into a prepubertal state. One year after treatment, $94 \%$ of the women were ovulating normally, the researchers reported at a conference in July.

However, because it was a proof-of-concept study, the trial did not include a control group. In their mid-20s, notes Oktay, many women

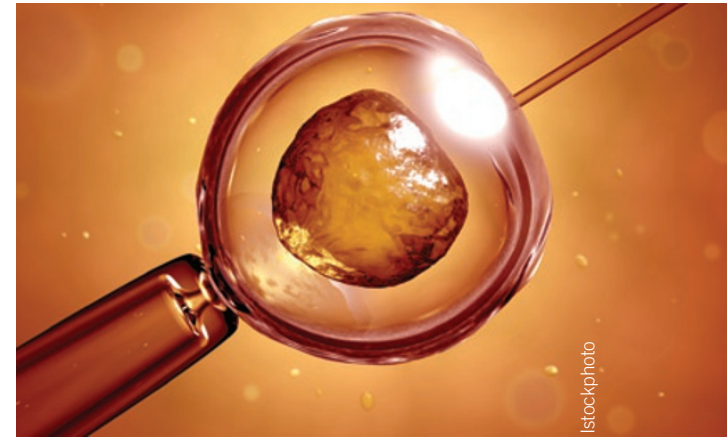

The magic moment: Fertilization of the egg

Conclusive evidence for the possible adverse effects of IVF is unavailable, owing to the dearth of long-term follow up of babies conceived through the technology. One Canadian study reported at the Society for Maternal-Fetal Medicine's conference in 2007 found that babies conceived through IVF were nearly $60 \%$ more likely to develop birth defects than naturally conceived ones. Most of the defects were gastrointestinal, although some were bone, muscle or heart related. Another study, from the University of Iowa, found birth defects in about $6.2 \%$ of about 1,500 IVF-conceived children, in contrast to $4.4 \%$ among naturally conceived ones (Fertil. Steril. 84, 1308-1315; 2005).

If future studies bear out these links, Williams suggests that one can perhaps decrease the risk to the child by avoiding certain invasive procedures that might not be necessary depending on individual circumstances, such as biopsies of implanted embryos, culturing embryos in the lab longer than the minimal time period and using ICSI in the absence of male fertility problems.

Prashant Nair, Chapel Hill, North Carolina do ovulate after chemotherapy, but their egg reserve is diminished, and they go into early menopause. It's too early to say whether this might also happen with the participants in the Australian trial. Stern says her team is set to launch a randomized clinical trial to test cetrorelix further.

Other candidate treatments, such as the compound sphingosine-1-phosphate, prevent the eggs from self-destructing in response to radiation, but research on those candidates has for the most part been limited to animal models. Pending further studies on these compounds, experts say the best option for preserving fertility in women is still to freeze embryos or eggs.

Apoorva Mandavilli, New York 